\title{
Watching rocks grow
}

\author{
JOHN VEYSEY II AND NIGEL GOLDENFELD* \\ Department of Physics, University of Illinois at Urbana-Champaign, 1110 W. Green St., Urbana, Illinois 61801, USA \\ *e-mail: nigel@uiuc.edu
}

Nature abounds with beautiful and striking landscapes, but a comprehensive understanding of their forms requires examples where detailed comparisons can be made between theory and experiment. Geothermal hot springs ${ }^{1}$ produce some of the most rapidly changing terrestrial landscapes, with reported travertine (calcium carbonate) growth rates as high as $5 \mathrm{~mm}$ per day $^{2-4}$. Unlike most landscapes, the patterns of which are the result of erosion processes on timescales of millions of years, the hot-spring depositional landscapes exhibit a spectacular cascade of nested ponds and terraces ${ }^{5}$, for which the origins and quantitative characterization have remained elusive. Here, we take advantage of this millionfold difference in geological timescale to present a novel combination of data from time-lapse photography, computer simulation and mathematical modelling that explains the emergence of the large-scale pond and terrace patterns, predicts and verifies the dynamics of their growth and shows that these patterns are scale invariant.

The dynamics of turbulent fluid flow coupled to the precipitation-driven growth of the travertine substrate was modelled on a discrete lattice of cells. Our cell dynamical system (CDS) is a set of rules, described in detail in the Methods section, that updates the lattice variables representing the heights of the landscape and fluid above each cell. The rules mimic fluid depositional dynamics and the influence of landscape features on the flow pattern ${ }^{6,7}$, enabling efficient computations of complex landscapes. Such a formalism complements analytic descriptions of carbonate precipitation patterns, such as our work on travertine domes $^{7}$ and recent studies of needle-like speleothem growth ${ }^{8,9}$. We were able to verify that our CDS model is quantitatively equivalent to the more conventional approach using differential equations, by using it to analyse the growth of single travertine domes $^{7}$. The CDS enables long-time (28,000 steps), large-scale $600 \times 500$ cells) simulations of landscapes with arbitrary, complex structure. Statistical properties were computed by averaging over 130 independent simulations. The model was not 'tuned': as long as the parameters are not varied so much that the model ceases to make physical sense, it produces the same morphological and statistical results, showing that our findings are generic for this class of precipitation patterns.

Figure 1 shows frames captured from a typical time-dependent simulation, initiated on a sloping plane with small initial roughness. Initial depositional instabilities grow to form dams, pond water pools behind the dams and complicated interactions arise as terraces grow and interact. The frames show a process of pond inundation, or 'drowning. In this mechanism, which seems to be the dominant one through which larger ponds form, the lip of a downstream pond grows more rapidly than that of its upstream neighbour. Eventually the downstream lip becomes taller, inundating the upstream pond, and leaving a single large pond. Although we also observed this mechanism in one-dimensional simulations, we obtain markedly different statistical results without the two lateral dimensions. Some aspects of pond growth have been simulated elsewhere ${ }^{10}$, using a simpler and less realistic precipitation model that ignores turbulent flow and capillary effects.

Our simulations also predict that, in areas of high flux, pond lips grow in the same direction as the flow. Although our precipitation model explicitly provides for increased growth rates in these areas, the direction of growth is not prescribed. In the simulations, this outward pond lip growth causes what geologists refer to as the 'apron and channel' facies ${ }^{4}$ to form immediately downstream from the vent. We observed these dynamics for a wide range of initial conditions, flow conditions and simulation parameters, strongly suggesting that these dynamics are generic and universal in hot-spring landscapes.

To test these predictions, we have undertaken a time-lapse photography project at the Mammoth Hot Spring complex in Yellowstone National Park, Wyoming, USA. For the past two years, we have collected still-camera photos at a carefully chosen location, which we have subsequently processed to produce movies of travertine growth. Figure 2 shows a series of four stills from a year-long time-lapse movie at Canary Springs. They confirm that pond inundation is a real process through which larger ponds are created. These stills also show some pond lips growing along with the flow, as was seen in simulation.

The dynamics of pond merging can be understood quantitatively by considering the formation of ponds of larger area as resulting solely from the aggregation of two smaller ponds. Simultaneous aggregation of three or more ponds is vanishingly unlikely. We characterize the statistical properties of the hot-spring landscape by the probability distribution of pond areas $P(A)$, which is proportional to the number of ponds of a given size, $n(A)$.

In a steadily evolving state at time, $t$, the number of ponds at a later time, $t+\delta t$, can be determined by merely counting the number of smaller ponds that combined to make a pond of size $A$. There are no further processes that create new ponds except at the smallest scale, which we discuss further below. Thus:

$$
n(A, t+\delta t)=\int_{1 / 2}^{1} n(f A, t) \mathrm{d} f .
$$

Here, $f$ is a fraction, $f \in(0,1]$. In this equation, a new pond of area $A$ can arise only when a smaller pond of size $f A$ merges with one of size $(1-f) A$, resulting in a single pond. It does not matter whether all smaller ponds merge, or just a fraction, because equation (1) is linear; if we assume that only a fixed fraction of ponds merge, the result does not change. Note that this integral only ranges from $f=1 / 2$ to $f=1$, which prevents overcounting. 


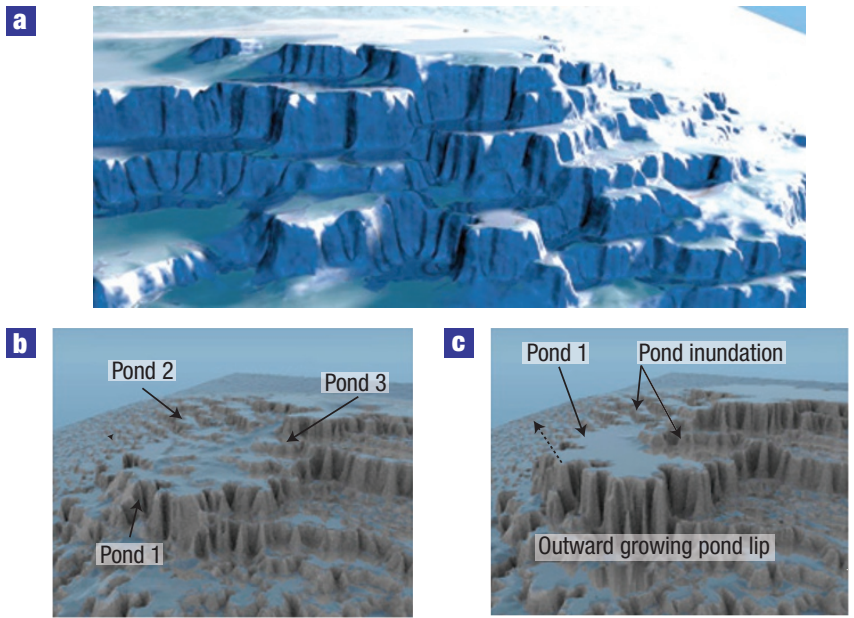

Figure 1 Dynamics of synthetic hot-spring landscapes. These still frames are rendered from CDS simulations. a, Overview of results after long simulation times. The model ultimately results in a well-developed landscape that is qualitatively very similar to those observed in the field. $\mathbf{b}$, A small part of the simulation at an early time. c, The same small region at a later time, showing the inundation of upstream ponds and the outward growth of pond lips, two growth mechanisms subsequently observed in time-lapse movies of real travertine deposition (see Fig. 2).

This formulation, however, omits an important detail: the smallest ponds have a non-zero size, and thus there should be a physically imposed cutoff on our description, below which new very small ponds arise and act as a boundary condition for the process of pond merger. If the smallest possible pond has size $\alpha$, then we must rewrite equation (1) as:

$$
n(A, t+\delta t)=\int_{1 / 2}^{1-\alpha / A} n(f A, t) \mathrm{d} f .
$$

The steady-state solution of integral equation (2) (see the Supplementary Information) predicts that for large ponds, the landscape is scale invariant, with the number $n(A)$ of ponds of area $A$ varying as $n(A) \sim A^{-2}$. Corrections arising from the non-zero minimum pond size lead to a slight modification of this prediction for small ponds $\alpha / A \rightarrow 1^{-}$, where $n(A) \sim A^{-2} /(1-\alpha / A)$. Hence, when $\alpha / A \lesssim 1$, the solution resembles a distribution with $z \gtrsim 2$, but is in fact not actually a pure power law.

We tested this prediction using both our CDS simulation and high-resolution photographs of small, centimetre-scale terraces taken from about $2 \mathrm{~m}$ high. By carefully tracing all of the features in these images (Fig. 3b), we computed the statistical properties of the photographed landscape, and compared them with simulation results. This methodology is further explained in the Supplementary Information. Although this only captures the smallest ponds, and none of the large-scale morphology that dominates the landscape, it nonetheless provides a basis for initial quantitative comparisons between simulation results and real landscapes. The log-log plot in Fig. 4a shows that the probability of finding a pond of a given area indeed follows a power law: $P(A) \propto A^{-b}$, with $b=-2.20 \pm 0.08$ (simulation) and $b=-2.29 \pm 0.54$ (photography). Owing to the relatively small pond sizes sampled, the effective power law is slightly greater than 2, in accord with expectations from the mathematical analysis above. The small number of large ponds in our data sets results in the significant uncertainty in these exponents. This uncertainty precludes meaningful estimation of
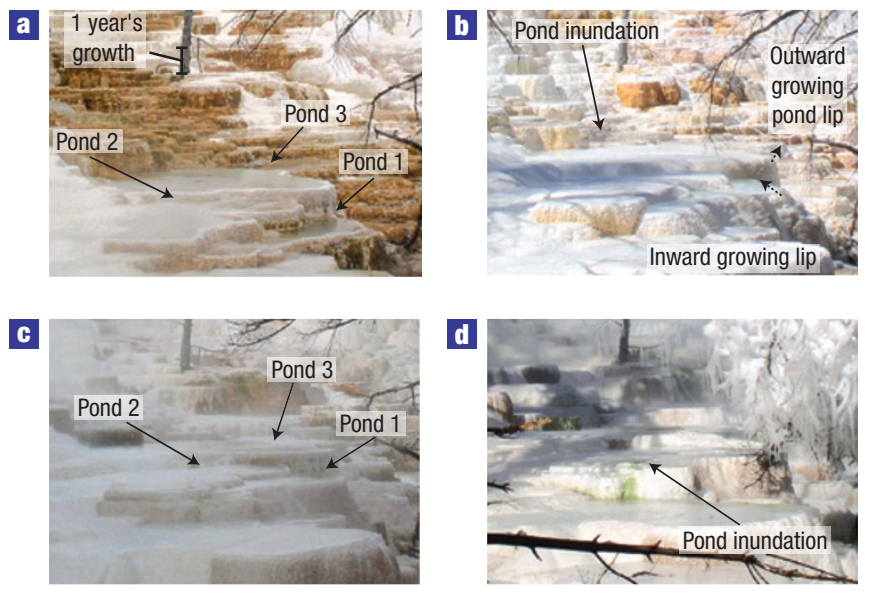

Figure 2 Watching rocks grow. Four still frames from our time-lapse movie, showing one year at Canary Springs. The frames are taken roughly three months apart, show the growth and interaction of several ponds and illustrate three specific processes. a, The initial state, together with the extent of subsequent precipitation. b, The first inundation of an upstream pond. c,d, After subsequent growth (c), the lip of the same pond is then inundated by its own downstream neighbour (d), forming a single large pond. This series demonstrates how large ponds form through the pairwise merging of smaller ones. Comparing frames $\mathbf{a}$ and $\mathbf{b}$ also shows how pond lips can grow into and out of the flow, possibly depending on the flux in the pond. Spring water flows from left to right in the photos. Photographs by Brian Suderman.

the cutoff, $\alpha$, which is a number smaller than both our numerical accuracy and observed pond areas. In spite of this, the observed exponents are remarkably close to those predicted by our crude theoretical analysis.

To quantify the shape of terraces, spatial variations in terrace shape and size, and temporal changes in macroscopic patterns, we compute from both simulations and photographs the 'terracewidth distribution' (TWD), analogous to the concept used fruitfully in surface science ${ }^{11}$. For present purposes, this is more useful than height correlation functions (for example, as used to characterize the layers in stromatolites ${ }^{12}$ ).

We define the terrace width by measuring the width $\lambda$ perpendicular to the 'maximum chord' in each pond. This is the longest chord that begins and ends on the edge a pond. This definition of $\lambda$ reduces to a terrace width in both an average sense and in the limit of an actual terrace, viewed as a very largeaspect-ratio pond. We define and measure the aspect ratio of a terrace, $\alpha$, as the ratio between the maximum chord and the largest terrace width. TWDs are usually characterized by the probability of finding a particular terrace width, $P(s)$, where $s=\lambda /\langle\lambda\rangle, \lambda$ is the actual terrace width and $\langle\lambda\rangle$ is the average of $\lambda$. For the purposes of our system, we calculate $\langle\lambda\rangle$ separately for each terrace or elongated pond.

Figure $4 \mathrm{~b}-\mathrm{d}$ shows the probability distribution of aspect ratio observed in both simulation and field data (Fig. 4b), the terrace width distribution $P(s)$ from both simulation and field data (Fig. 4c) and the asymptotics of $P(s)$ as predicted analytically and compared with simulation results (Fig. $4 \mathrm{~d}$ ). The analytic predictions were made on the basis of a standard statistical mechanical model that approximates the terrace configurations as the world lines of free fermions in one space and one time dimension ${ }^{13}$ to yield $P_{\mathrm{ff}}(s)$.

In such a model, particles execute a random walk along the $x$ axis, and trace out a set of trajectories in space-time that cannot 

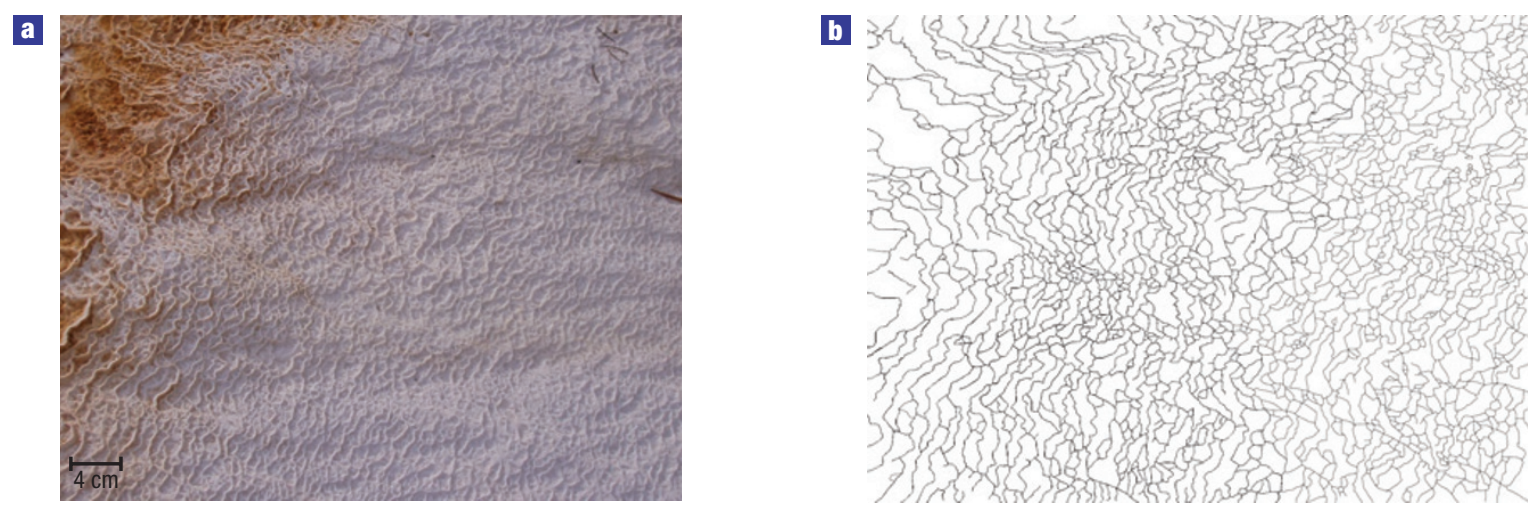

Figure 3 Tracing out the data. a, Photograph of small terraces at Spring AT-3. b, Pak Yuen Chan's painstaking tracing of the lips in the photo. We digitized this tracing and compared it with simulation results.

a

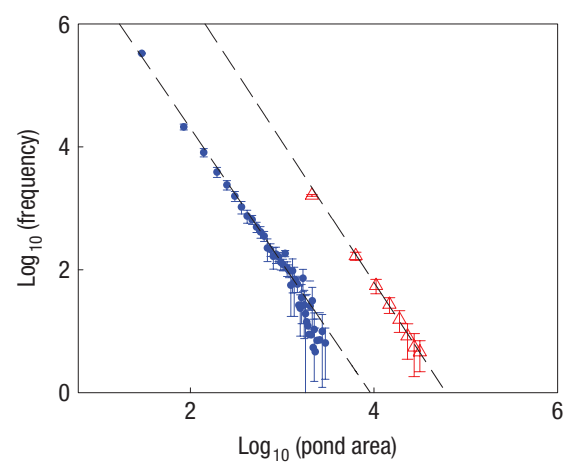

G

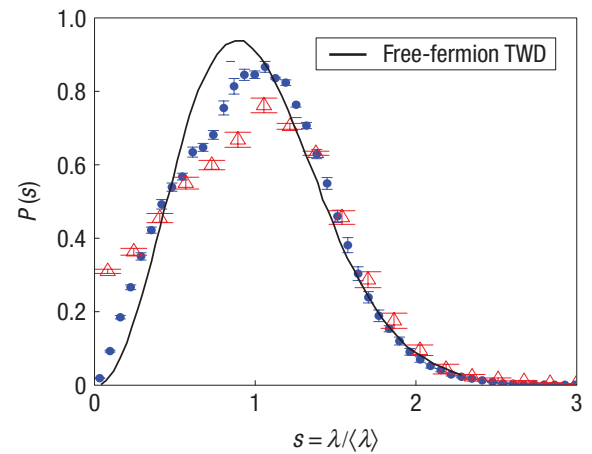

b

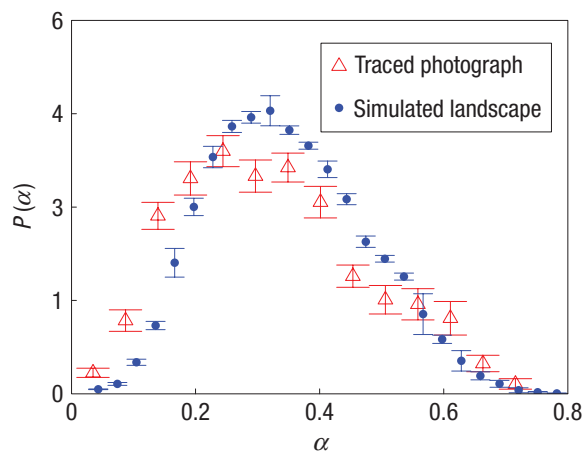

d

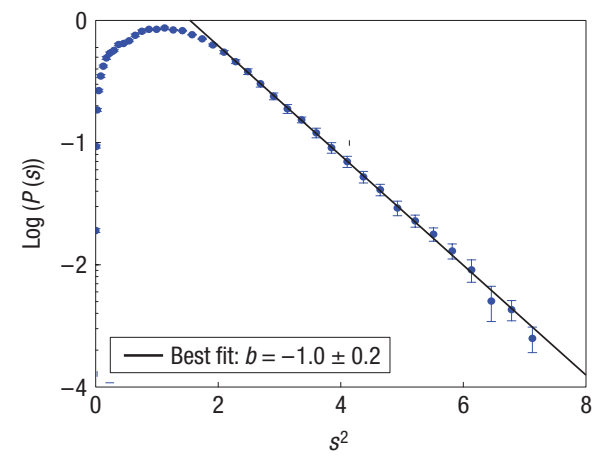

Figure 4 Static statistical properties of travertine landscapes. Data from 130 simulated landscapes each of which began from a sloping plane with different random noise added. Best fit lines are shown, and horizontal uncertainty is shown by the width of the vertical error bars, which denote one standard deviation. a, Log-log plot showing the frequency of occurrence of a given pond area. $\mathbf{b}$, The distribution of pond aspect ratios, $\alpha$, comparing simulations with the digitized photograph. $\mathbf{c}$, The TWD for simulations compared with both experimental observations and the TWD predicted by the free-fermion model of Joós ${ }^{13}$. d, At large $s, P(s) \sim \mathrm{e}^{-s^{2}}$, implying that effective interactions in our system are short range. $b$ is the slope of the fit line.

cross. The fermion constraint mimics the fact that terraces are at different heights and so never intersect. This stochastic process provides a satisfactory account of terrace morphology for miscut vicinal crystal surfaces ${ }^{13}$, and our use of it here is an uncontrolled first approximation that ignores ponding on short scales, but is a generic description at long scales, as we show below.

For $s>1.5$, the hot-spring TWD is well approximated by $P_{\mathrm{ff}}(s)$, and in this limit can be shown to be equivalent to that of a random walking particle; hence $P(s)$ has a gaussian tail (with logarithmic corrections $)^{13}$. On a plot of $\log _{10} P(s)$ versus $s^{2}$, this asymptotic behaviour should appear as a straight line, and indeed the simulations follow this prediction very well.

This implies that any interactions between terrace lips are short range, and that at large $s$ the lips do not interact. Furthermore, the deviations from the free-fermion distribution at small $s$ enable us to infer the nature of the effective interactions in our system. $P(s)>P_{\mathrm{ff}}(s)$ for $s<0.5$ implies an effective attraction between the edges of terraces. 


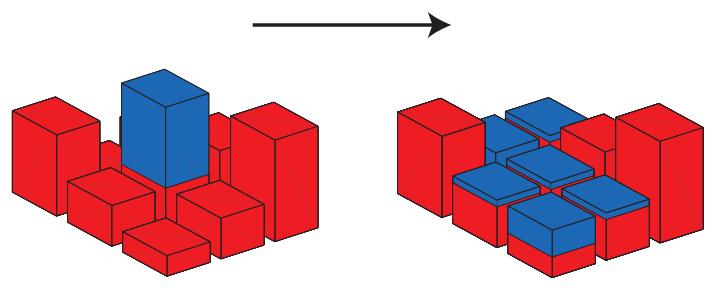

Figure 5 Schematic diagram of one time step in the cellular model. Each cell in the model interacts with its eight nearest neighbours. Here, the water originally located in the centre cells forms a locally flat surface by spreading to five neighbouring cells.

Even though the microscopic description of travertine precipitation depends on myriad biological, chemical and physical details, we can predict the emergence of terrace patterns on large spatial scales from a minimal model of fluid flow and irreversible deposition.

'Watching rocks grow' enabled us to test the minimal model's key predictions about the dynamics of landscape evolution, leading to an analytical description of terrace formation and predictions of the hot-spring landscape's statistical properties that are in concordance with field data. Although it is essential to test these predictions further through comparison with real hot springs, we anticipate that this quantitative and novel methodology can be replicated in other complex pattern-forming systems with sufficiently rapid growth dynamics, such as at hydrothermal vents ${ }^{14}$.

\section{METHODS}

In the cellular model, we represent the quantities of interest as cells on a lattice, and the landscape as stacked 'bricks' on top of which water flows ${ }^{7}$. This is schematically shown in Fig. 5 . Each lattice site, indexed by $i$, contains the following quantities: landscape height $H_{i}$, water depth $W_{i}$, temperature $T_{i}$, calcium concentration $C_{i}$ and $\mathrm{CO}_{2}$ concentration $V_{i}$. There are four rules that govern the interactions between neighbouring cells during each simulation time step. These rules are 'caricatures' of real processes rather than a discretization of any partial differential equation.

The first rule controls how water is added to the simulation. Water is added with specified initial chemistry $\left(C_{0}, V_{0}, T_{0}\right)$ at the spring source $(i=0): W^{\prime}(0)+H(0)=W_{0}+H_{0}(0)$. Here, $H_{0}(0)$ is the landscape height when the simulation began. This rule reflects water being added at constant pressure (initially an amount $W_{0}$ ), implying the simulation must eventually stop. In addition, local chemistry changes proportionally: $C^{\prime}(0)=\left(C(0) W(0)+\delta W(0) C_{0}\right) / W_{0}$ and similarly for $V^{\prime}(0)$ and $T^{\prime}(0)$.

The second rule governs fluid transport, which occurs much faster than deposition, and is done in a way that allows ponding and accounts for turbulent effects. Defining $H^{T} \equiv H+W, S_{i, j} \equiv H_{i}-H_{j}^{T}$, and a sum over nearest neighbours, $N_{i} \equiv \sum_{S_{i, j}>0} S_{i, j}$, there are two cases to consider. If $N_{i}>W_{i}$, flow is in accord with Chézy's law, and the flux $Q_{i, j}=W_{i} / \sqrt{S_{i, j}} / N_{i}$. If $N_{i}<W_{i}$, water is flattened locally, as in the schematic diagram in Fig. 5 . In both cases, moving fluid advects local chemistry.

The third rule controls how water chemistry changes to reflect slope-dependent $\mathrm{CO}_{2}$ outgassing $\left(V_{j}^{\prime} \propto \sqrt{S_{i, j}} /\left(1+\sqrt{S_{i, j}}\right)\right.$, decreasing temperature $\left(T^{\prime} \propto T\right)$ and the overall precipitation reaction $\mathrm{Ca}^{2+}+2 \mathrm{HCO}_{3}^{1-} \rightleftharpoons \mathrm{CO}_{2}+\mathrm{CaCO}_{3}+\mathrm{H}_{2} \mathrm{O}$.

The fourth and final rule governs precipitation, a process that depends on the local surface geometry. Defining a crude potential to precipitate, $\Omega \equiv C-V$, the height changes according to: $\delta H_{j} \propto \Omega_{i}\left(R_{1}+R_{2} \mathbf{Q}_{i, j} \cdot \hat{n}_{i, j}+R_{3} \sqrt{S_{i, j}}\right)$. Here $R_{1}$ is a small constant, the second term reflects precipitation proportional to the flux normally incident on the surface, and the last term accounts for increased precipitation due to Bernoulli effects and local degassing.

Simulations were started on an inclined plane with a slope of 5.71 degrees, so that the elevation was 50 over a horizontal distance of 500 cells. Small random perturbations of height between 0.07 and -0.07 were added to seed initial instabilities. The results presented in this manuscript are based on 130 simulations with 28,000 time steps, and using subpixel interpolation to measure properties of the simulated landscape.

To estimate the error bars and to better calculate the distributions shown in Fig. 4, we used the bootstrap method ${ }^{15,16}$, resampling our original set of measurements with replacement, to obtain a synthetic data set with the same number of measurements. This new data set contains duplicate measurements (hence the requirement to sample with replacement). We then calculated the distribution function based on the synthetic data set. We repeated this process 1,000 times for the simulation results and 10,000 times for the smaller data set that came from the digitized photograph. From the distribution of each point in the resulting histograms, we then calculated the mean and standard deviation; these are our best estimates and the error in those estimates. All distributions were calculated in the same manner. We also note that any bins with less than three counts were considered to have insufficient data, and are not plotted.

The uncertainty obtained through the bootstrap method was propagated in the usual manner as we calculated the normalized distribution $P(s)$, a step needed to compare the two distributions. Note that there is also a horizontal uncertainty, due to the bin size, which is represented by the width of the top and bottom of the error bars. The overall agreement between these curves is quite good, although the smallest data point in the experimental curve deviates from the simulation predictions, an effect probably due to our inability to resolve very small details in the traced terrace edges.

\section{Received 20 June 2007; accepted 4 February 2008; published 16 March 2008.}

\section{References}

1. Ford, T. \& Pedley, H. A review of tufa and travertine deposits of the world. Earth Sci. Rev. 41 $117-175$ (1996).

2. Friedman, I. Some investigations of the deposition of travertine from hot springs: I. The isotope chemistry of a travertine-depositing spring. Geochim. Cosmochim. Acta 34, 1303-1315 (1970).

3. Pentecost, A. \& Viles, H. A. A review and reassessment of travertine classification. Geogr. Phys. Quaternaire 48, 305-314 (1994).

4. Fouke, B. W. et al. Depositional facies and aqueous-solid geochemistry of travertine-depositing hot springs (Angel Terrace, Mammoth Hot Springs, Yellowstone National Park, USA). J. Sed. Res. 70, 265-285 (2000)

5. Allen, E. T. \& Day, A. L. Hot springs of the Yellowstone National Park. Tech. Rep. publication 466, Carnegie Institute of Washington (1935).

6. Murray, A. B. \& Paola, C. A cellular model of braided rivers. Nature 371, 54-57 (1994).

7. Goldenfeld, N., Chan, P. \& Veysey, J. Dynamics of precipitation pattern formation at geothermal hot springs. Phys. Rev. Lett. 96, 254501 (2006).

8. Short, M. B., Baygents, J. C. \& Goldstein, R. E. Stalactite growth as a free-boundary problem. Phys. Fluids 17, 083101-083112 (2005).

9. Short, M. B. et al. Stalactite growth as a free-boundary problem: A geometric law and its platonic ideal. Phys. Rev. Lett. 94, 018501-018504 (2005).

10. Hammer, Ø., Dysthea, D. \& Jamtveit, B. The dynamics of travertine dams. Earth Planet. Sci. Lett. 256, 258-263 (2007)

11. Jeong, H.-C. \& Williams, E. D. Steps on surfaces: Experiment and theory. Surf. Sci. Rep. 34, 171-294 (1999).

12. Grotzinger, J. P. \& Rothman, D. H. An abiotic model for stromatolite morphogenesis. Nature 383 423-425 (1996).

13. Joós, B., Einstein, T. L. \& Bartelt, N. C. Distribution of terrace widths on a vicinal surface within the one-dimensional free-fermion model. Phys. Rev. B 43, 8153-8162 (1991).

14. Stone, D. \& Goldstein, R. Tubular precipitation and redox gradients on a bubbling template. Proc. Natl Acad. Sci. 101, 11537-11541 (2004).

15. Efron, B. \& Tibshirani, R. An Introduction to the Bootstrap (Chapman \& Hall/CRC, New York, 1994).

16. Press, W. H., Teukolsky, S. A., Vetterling, W. T. \& Flannery, B. P. Numerical Recipes in C 2nd edn (Cambridge Univ. Press, New York, 1992).

\section{Acknowledgements}

This work was carried out as part of a comprehensive geochemical and microbial ecology study of Yellowstone's carbonate hot springs. We thank our colleagues B. Fouke, M. Kandianis and T. Schickel for valuable discussions. A special debt is due to ranger B. Suderman of the US Park Service for photography, N. Guttenberg for assistance rendering simulations and P. Y. Chan for discussions and penmanship. This work was funded by the US National Science Foundation.

Correspondence and requests for materials should be addressed to N.G.

Supplementary Information accompanies this paper on www.nature.com/naturephysics.

\section{Author contributions}

J.V. and N.G. contributed equally to all aspects of this research.

Reprints and permission information is available online at http://npg.nature.com/reprintsandpermissions/ 